\title{
PHYSIOLOGICAL REACTION OF BASKET WILLOW (Salix viminalis L.) TO ZINC EXCESS
}

\section{REAKCJA FIZJOLOGICZNA WIERZBY WICIOWEJ (Salix viminalis L.) NA NADMIAR CYNKU}

Department of Plant Physiology and Biochemistry, West Pomeranian University

of Technology, Szczecin, Poland

\begin{abstract}
Streszczenie. Badano wpływ soli cynku o stężeniu $50-650 \mathrm{mg} \cdot \mathrm{dm}^{-3}$ na reakcję fizjologiczną wierzby wiciowej, odmian Bjor, Tora i Jorr, uprawianej w hydroponice. Oznaczono zawartość barwników asymilacyjnych, wskaźniki względnej zwartości wody i deficytu wysycenia wodą, intensywność procesów asymilacji i transpiracji, przewodność szparkową, fotosyntetyczny współczynnik wykorzystania wody (WUE) oraz chwilowy fotosyntetyczny współczynnik wykorzystania wody (WUEI). Stwierdzano spadek natężenia badanych parametrów fizjologicznych wraz ze wzrostem dawki soli cynku w pożywce. Asymilacja i transpiracja wierzby wiciowej były w istotny sposób ograniczone szparkowo. Dodatek soli cynku do pożywki spowodował wzrost deficytu wysycenia wodą liści badanych odmian wierzby.
\end{abstract}

Key words: gas exchange parameters, photosynthetic pigments, water use efficiency, common osier, zinc.

Słowa kluczowe: parametry wymiany gazowej, barwniki asymilacyjne, współczynniki wykorzystania wody, wierzba wiciowa, cynk.

\section{INTRODUCTION}

Pollution of the natural environment requires taking intensive efforts aimed at restoring its initial state. Among many pollutants that adversely affect the environment are, among others, heavy metals. One of them is zinc which is an element essential for plants but at the same time very toxic if present in excess in the environment. It belongs to the group of the most mobile heavy metals (Baran and Jasiewicz 2009). It activates many enzymes in a plant, is a component of reduced NAD (nicotinamide adenine dinucleotide) dehydrogenase and NADP (nicotinamide adenine dinucleotide phosphate), peptidase, and phosphorylase. Zinc regulates the proportions of components at the cell level, which affects the permeability of cell membranes (Szatanik-Kloc and Bowanko 2007; Szatanik-Kloc et al. 2010). In addition, it participates in the process of photosynthesis and the metabolism of phosphorus compounds, carbohydrates and proteins. It also belongs to the group of metals that affect the synthesis of auxins (Kabata-Pendias and Pendias 1999).

Corresponding author - Adres do korespondencji: Katarzyna Malinowska, Department of Plant Physiology and Biochemistry, West Pomeranian University of Technology, Szczecin, Juliusza Słowackiego 17, 71-434 Szczecin, Poland, e-mail: katarzyna.malinowska@zut.edu.pl 
Zinc toxicity involves reduction of the most important life process in plants, both at the physiological and biochemical level (Gruca-Królikowska and Wacławek 2006) and reduces the size of leaf blades and inhibits the growth of plant roots (Kostrzewska-Kuczumow and Weryszko-Chmielewska 2000a, b).

The efficiency and duration of reclamation using phytoremediation depends not only on the concentration of pollutants in substrate but also on the proper selection of a plant species to a given type of contamination (Baran 2000; Zemleduch and Tomaszewska 2007). Studies conducted in the field of chemistry, physiology or biochemistry have allowed for a more accurate understanding of the mechanisms and processes being responsible for the uptake and accumulation of toxins by plants, as well as using the physiological and biochemical parameters to assess the suitability of plants for reclamation of degraded lands. This makes it possible to intentionally use natural capabilities of plants and plan optimal methods of pollutant remediation from a degraded land (Zemleduch and Tomaszewska 2007).

One of the methods of reclamation of zinc-contaminated land is phytoremediation. In terms of phytoremediation of large industrial protection zones and urban areas, this is the basket willows, many species of which are considered to be resistant to soil contamination with heavy metals, that seem particularly predisposed for this purpose (Landberg and Greger 1996; Laureysens et al. 2004; Szczukowski et al. 2004; Laureysens et al. 2005; Wrzosek et al. 2008; Zabrocki and Ignacek 2008). In the assessment of the suitability of plants for reclamation of degraded land, their physiological and biochemical parameters are used.

This study aimed at comparing three cultivars of the basket willow in terms of their physiological activity, i.e. assimilation and transpiration rates, stomatal conductance, photosynthetic pigments, photosynthetic water-use efficiency, photosynthetic intrinsic water-use efficiency, relative water content and water saturation deficit on the increased content of zinc ions in nutrient solution.

Verification of the willow as a particularly valuable genus for phytoremediation, understanding of the physiological foundations of its resistance to stress induced by the increased content of zinc in nutrient solution will help pre-determine the suitability of these cultivars for phytoremediation.

\section{MATERIAL AND METHODS}

Three cultivars of the basket willow (Salix viminalis L.), namely Bjor, Jorr and Tora, were used as the experimental material. The study was performed on 180 willow cuttings obtained from one-year shoots. Laboratory analyses were carried out on the plant material from aerated hydroponics with different doses of zinc salt. The willow cuttings, divided into four groups of equal numbers and placed in hydroponics filled with complete Hoagland nutrient solution. Zinc sulphate was added to nutrient solution 14 days after placing the willow cuttings in hydroponics, after plant rooting and after shoot development. Three levels of nutrient solution contamination with zinc salt were applied: 50,250 and $650 \mathrm{mg} \cdot \mathrm{dm}^{-3}$. The control object was the plants placed in complete nutrient solution according to Hoagland. Determination of physiological parameters was performed in three times: on day 7, 14 and 21 from the application of zinc salt doses. Measurements of gas exchange parameters $\left(\mathrm{CO}_{2}\right.$ 
assimilation, transpiration and stomatal conductance) were performed on leaves (repeating the measurement five times), using a PP Systems gas exchange analysis system TPS-2 (Amesbury, MA, USA), operating in open-system configuration with PLC-4 broad style leaf cuvette. The following conditions were used in the cuvette: a constant supply of carbon dioxide, humidity equal to ambient humidity and illumination equal to $2053 \mathrm{PAR}\left(\mu \mathrm{mol} \cdot \mathrm{m}^{-2} \cdot \mathrm{s}^{-1}\right)$, being provided by a light unit attached to the cuvette.

Based on the results of assimilation and transpiration rates and stomatal conductance, photosynthetic water-use efficiency (WUE) and photosynthetic intrinsic water-use efficiency (WUEI) were calculated. Determination of the photosynthetic pigments (chlorophyll-a and chlorophyll-b and carotenoids) was conducted on the same leaves on which gas exchange parameters were determined. The content of chlorophyll was determined by the method of Arnon et al. (1956) as modified by Lichtenthaler (1987), whereas the content of carotenoids by the method of Hager and Meyer-Bethenrath (1966). Relative water content (RWC) and water saturation deficit (WSD) were determined according to Bandurska (1991). Statistical analysis was performed using the Duncan's test, at the significance level $\operatorname{LSD}_{0.05}$. The results were processed using Statistica 10 software package. The coefficients of Pearson's linear correlation $(r)$ were calculated between the analysed variables that characterise gas exchange.

\section{RESULTS AND DISCUSSION}

The findings showed a variation in the physiological parameters being determined, depending on the basket willow cultivar and the zinc salt dose.

Statistical analysis showed significant differences in the content of photosynthetic pigments in the examined basket willow cultivars depending on the zinc salt dose. The highest dose of zinc salt being used induced reduction in the level of chlorophyll-a by $54.8 \%$, $53.4 \%$ and $50.2 \%$ and that of chlorophyll-b by $54.0 \%, 53.5 \%$ and $49.4 \%$ in three basket willow cultivars Bjor, Jorr and Tora, respectively (Table 1). The content of carotenoids in the basket willow cultivars Bjor, Jorr and Tora using a zinc salt dose of $650 \mathrm{mg} \cdot \mathrm{dm}^{-3}$ was $53.2 \%$, $46.8 \%$ and $47.9 \%$, respectively, of their content in the control plant leaves (Table 1). The average values of the content of photosynthetic pigments ranged from 701 to $1964\left(\mu \mathrm{g} \cdot \mathrm{g}^{-1} \mathrm{FM}\right)$ for chlorophyll-a, 270 to 810 ( $\mu \mathrm{g} \cdot \mathrm{g}^{-1} \mathrm{FM}$ ) for chlorophyll-b, and 386 to 919 ( $\mu \mathrm{g} \cdot \mathrm{g}^{-1} \mathrm{FM}$ ) for carotenoids (Table 1). Statistical analysis did not show any differences in the content of photosynthetic pigments between the basket willow cultivars being examined (Table 1). The reduced contents of chlorophylls and carotenoids in the same basket willow cultivars using different doses of heavy metals were obtained by Malinowska et al. (2010a, b, 2011, 2015); Malinowska and Wróbel (2015).

The indicator of changes in the water balance in a plant is, among others, relative water content (RWC) and water saturation deficit (WSD). Significant differences in the RWC and WSD values were obtained depending on the zinc salt dose being used. Increasing doses of zinc salt induced reduction in the water content (RWC) in leaves of the examined basket willow cultivars. The highest dose of zinc salt being used in nutrient solution induced an increase in water saturation deficit (WSD) in three basket willow cultivars Bjor, Jorr and Tora by $9.8 \%$, $6.5 \%$ and $15.5 \%$, respectively, in relation to the same cultivars of the control object (Table 1 ). 
Table 1. Mean values of chloroplast pigments and water indices for interaction between variety basket willow and dose of zinc salt, and for the primary effects

Tabela 1. Średnie wartości barwników asymilacyjnych oraz wskaźniki bilansu wodnego dla interakcji między odmianami wierzby wiciowej a dawką soli cynku oraz dla efektów głównych

\begin{tabular}{|c|c|c|c|c|c|c|}
\hline $\begin{array}{c}\text { Variety } \\
\text { Odmiana }\end{array}$ & $\begin{array}{c}\text { Dose } \\
\text { Dawka } \\
{\left[\mathrm{mg} \cdot \mathrm{dm}^{-3}\right]}\end{array}$ & $\begin{array}{c}\text { Chlorophyll a } \\
\text { (\% of control - } \\
\text { kontroli) } \\
\text { Chlorofil a } \\
{\left[\mu \mathrm{g} \cdot \mathrm{g}^{-1} \mathrm{FW}\right]}\end{array}$ & $\begin{array}{l}\text { Chlorophyll b } \\
\text { (\% of control - } \\
\text { kontroli) } \\
\text { Chlorofil b } \\
{\left[\mu \mathrm{g} \cdot \mathrm{g}^{-1} \mathrm{FW}\right]}\end{array}$ & $\begin{array}{c}\text { Carotenoids } \\
\text { (\% of control - } \\
\text { kontroli) } \\
\text { Karotenoidy } \\
{\left[\mu \mathrm{g} \cdot \mathrm{g}^{-1} \mathrm{FW}\right]}\end{array}$ & $\begin{array}{c}\text { RWC } \\
{[\%]}\end{array}$ & $\begin{array}{c}\text { WSD } \\
{[\%]}\end{array}$ \\
\hline \multirow{4}{*}{ Bjor } & 0 & $\begin{array}{c}1964 \pm 239 \mathrm{k} \\
(100)\end{array}$ & $\begin{array}{c}810 \pm 106 \mathrm{j} \\
(100)\end{array}$ & $\begin{array}{c}919 \pm 173 \mathrm{j} \\
(100)\end{array}$ & $89.6 \mathrm{~cd}$ & $10.4 \mathrm{~cd}$ \\
\hline & 50 & $\begin{array}{c}1238 \pm 109 h \\
(63.0)\end{array}$ & $\begin{array}{c}518 \pm 127 \mathrm{~g} \\
(63.9)\end{array}$ & $\begin{array}{c}604 \pm 114 \mathrm{~g} \\
(65.7)\end{array}$ & 87.2 bc & $12.8 \mathrm{ad}$ \\
\hline & 250 & $\begin{array}{c}1001 \pm 131 \mathrm{e} \\
(50.9)\end{array}$ & $\begin{array}{c}402 \pm 98 \mathrm{~d} \\
(49.7)\end{array}$ & $\begin{array}{c}572 \pm 101 \mathrm{f} \\
(62.2)\end{array}$ & $84.9 a b$ & $15.1 \mathrm{ab}$ \\
\hline & 650 & $\begin{array}{c}888 \pm 122 a \\
(45.2)\end{array}$ & $\begin{array}{c}378 \pm 103 b \\
(46.6)\end{array}$ & $\begin{array}{c}489 \pm 92 \mathrm{~d} \\
(53.2)\end{array}$ & $79.8 \mathrm{~g}$ & $20.2 \mathrm{e}$ \\
\hline \multirow{4}{*}{ Jorr } & 0 & $\begin{array}{c}1407 \pm 186 \mathrm{i} \\
(100)\end{array}$ & $\begin{array}{c}588 \pm 116 \mathrm{~h} \\
(100)\end{array}$ & $\begin{array}{c}825 \pm 142 \mathrm{i} \\
(100)\end{array}$ & $90.8 \mathrm{~d}$ & $9.2 \mathrm{c}$ \\
\hline & 50 & $\begin{array}{c}1030 \pm 152 \mathrm{f} \\
(73.2)\end{array}$ & $\begin{array}{c}442 \pm 108 \mathrm{e} \\
(75.2)\end{array}$ & $\begin{array}{c}504 \pm 131 \mathrm{e} \\
(61.2)\end{array}$ & $86.4 \mathrm{ab}$ & $13.6 \mathrm{ab}$ \\
\hline & 250 & $\begin{array}{c}767 \pm 117 d \\
(54.5)\end{array}$ & $\begin{array}{c}331 \pm 86 a \\
(56.2)\end{array}$ & $\begin{array}{c}468 \pm 138 a \\
(56.7)\end{array}$ & $84.9 a b$ & $15.1 \mathrm{ab}$ \\
\hline & 650 & $\begin{array}{c}701 \pm 126 \mathrm{~b} \\
(49.8)\end{array}$ & $\begin{array}{c}270 \pm 91 \mathrm{c} \\
(46.0) \\
\end{array}$ & $\begin{array}{c}386 \pm 85 b \\
(46.8)\end{array}$ & $84.3 \mathrm{a}$ & $15.7 \mathrm{~b}$ \\
\hline \multirow{4}{*}{ Tora } & 0 & $\begin{array}{c}1586 \pm 254 \mathrm{j} \\
(100)\end{array}$ & $\begin{array}{c}638 \pm 124 \mathrm{i} \\
(100)\end{array}$ & $\begin{array}{c}935 \pm 145 \mathrm{k} \\
(100)\end{array}$ & $86.3 a b$ & $13.7 \mathrm{ab}$ \\
\hline & 50 & $\begin{array}{c}1108 \pm 206 \mathrm{~g} \\
(69.9)\end{array}$ & $\begin{array}{c}500 \pm 153 \mathrm{f} \\
(78.3)\end{array}$ & $\begin{array}{c}624 \pm 117 \mathrm{~h} \\
(66.8)\end{array}$ & $85.1 \mathrm{ab}$ & $14.9 \mathrm{ab}$ \\
\hline & 250 & $\begin{array}{c}894 \pm 211 \mathrm{a} \\
(56.4)\end{array}$ & $\begin{array}{c}383 \pm 94 b \\
(60.0)\end{array}$ & $\begin{array}{c}467 \pm 121 \mathrm{a} \\
(49.9)\end{array}$ & $75.5 \mathrm{f}$ & $24.5 \mathrm{f}$ \\
\hline & 650 & $\begin{array}{c}737 \pm 189 \mathrm{c} \\
(46.5)\end{array}$ & $\begin{array}{c}323 \pm 76 \text { a } \\
(50.6)\end{array}$ & $\begin{array}{c}448 \pm 102 \mathrm{c} \\
(47.9)\end{array}$ & 70.8 e & $29.2 \mathrm{~g}$ \\
\hline \multicolumn{7}{|c|}{ Means for primary effects - Średnie dla efektów głównych } \\
\hline \multirow{3}{*}{$\begin{array}{l}\text { Varieties } \\
\text { Odmiana }\end{array}$} & Bjor & 1273 a & 527 a & 646 a & $85.4 \mathrm{a}$ & $14.6 \mathrm{a}$ \\
\hline & Jorr & 976 a & 408 a & 546 a & $86.6 \mathrm{a}$ & $13.4 \mathrm{a}$ \\
\hline & Tora & 1081 a & $461 \mathrm{a}$ & 619 a & $79.4 \mathrm{~b}$ & $20.6 \mathrm{~b}$ \\
\hline \multirow{4}{*}{$\begin{array}{l}\text { Dose } \\
\text { Dawka }\end{array}$} & 0 & $1652 \mathrm{c}$ & $679 \mathrm{c}$ & $893 d$ & $88.9 \mathrm{~b}$ & $11.1 \mathrm{a}$ \\
\hline & 50 & $1125 b$ & $487 \mathrm{~b}$ & 577 c & $86.2 \mathrm{~b}$ & $13.8 \mathrm{a}$ \\
\hline & 250 & 887 a & $372 \mathrm{a}$ & $502 \mathrm{~b}$ & $81.7 \mathrm{a}$ & $18.3 \mathrm{~b}$ \\
\hline & 650 & $775 a$ & $324 \mathrm{a}$ & $441 \mathrm{a}$ & $78.3 \mathrm{a}$ & $21.7 b$ \\
\hline
\end{tabular}

RWC - relative water content - wskaźnik względnej zawartości wody.

WSD - water saturation deficyt - wskaźnik deficytu wysycenia wodą.

$a-i-$ homogeneous groups according to the Duncana test, $\alpha<0.05$ - grupy jednorodne wyznaczone przy użyciu testu Duncana, $\alpha<0,05$.

Statistical analysis showed that from among the cultivars being examined, cultivar Tora was characterised by lowest values of relative water content (RWC) and water saturation deficit (WSD) compared to cultivars Bjor and Jorr (Table 1). The increased water saturation deficit (WSD) in the basket willow cultivars Bjor, Jorr and Tora after using different doses of heavy metals was obtained by Malinowska et al. (2010a, b, 2015), Malinowska and Wróbel (2015).

As the dose of zinc salt in nutrient solution increased, a decrease in net assimilation rate and transpiration rate was observed. The intensity of net assimilation process in cultivars Bjor, Jorr and Tora in the presence of the highest dose of zinc salt $\left(650 \mathrm{mg} \cdot \mathrm{dm}^{-3}\right)$ in nutrient solution was $53.9 \%, 61.4 \%$ and $52.9 \%$, respectively, in relation to the intensity of this 
process in the control plants (Table 2). Net assimilation rate in the examined basket willow cultivars growing in nutrient solution with a dose of zinc salt of $250 \mathrm{mg} \cdot \mathrm{dm}^{-3}$ decreased by $8.4 \%$ in cultivar Bjor, $10.6 \%$ in cultivar Jorr, and $14.7 \%$ in cultivar Tora in relation to the intensity of this process in the cultivars growing in complete nutrient solution without zinc salt added (Table 2). Statistical analysis showed that from among the basket willow cultivars being examined, cultivar Tora was characterised by a more intense net assimilation rate compared to cultivars Bjor and Jorr (Table 2).

Table 2. Mean values of gas exchange parameters and photosynthetic index of water use efficiency (WUE) and instantaneous photosynthetic index of water use efficiency (WUEI) for interaction between variety basket willow and dose of zinc salt, and for the primary effects

Tabela 2. Średnie wartości parametrów wymiany gazowej oraz fotosyntetyczny współczynnik wykorzystania wody (WUE) i chwilowy fotosyntetyczny współczynnik wykorzystania wody (WUEI) dla interakcji między odmianami wierzby wiciowej a dawką soli cynku oraz dla efektów głównych

\begin{tabular}{|c|c|c|c|c|c|c|}
\hline $\begin{array}{l}\text { Variety } \\
\text { Odmiana }\end{array}$ & $\begin{array}{c}\text { Dose } \\
\text { Dawka } \\
{\left[\mathrm{mg} \cdot \mathrm{dm}^{-3}\right]}\end{array}$ & $\begin{array}{l}\mathrm{CO}_{2} \text { assimilation } \\
\text { Asymilacja } \mathrm{CO}_{2} \\
(\% \text { of control - } \\
\quad \text { kontroli) } \\
{\left[\mu \mathrm{mol} \mathrm{CO}_{2} \cdot \mathrm{m}^{-2} \cdot \mathrm{s}^{-1}\right]}\end{array}$ & $\begin{array}{l}\text { Transpiration } \\
\text { Transpiracja } \\
\text { (\% of control - } \\
\text { kontroli) } \\
\left.\mathrm{mmol} \mathrm{H}_{2} \mathrm{O} \cdot \mathrm{m}^{-2} \cdot \mathrm{s}^{-1}\right]\end{array}$ & $\begin{array}{c}\text { Stomatal } \\
\text { conductance } \\
\text { Przewodność } \\
\text { szparkowa } \\
(\% \text { of control - } \\
\text { kontroli) } \\
{\left[\mathrm{mmol} \mathrm{H} \mathrm{H}_{2} \mathrm{O} \cdot \mathrm{m}^{-2} \cdot \mathrm{s}^{-1}\right]}\end{array}$ & $\begin{array}{l}\text { WUE } \\
\text { [umol C } \\
\mathrm{H}_{2}\end{array}$ & $\begin{array}{l}\text { WUEl } \\
\mathrm{O}_{2} \cdot \mathrm{mmol} \\
\left.\mathrm{O}^{-1}\right]\end{array}$ \\
\hline \multirow{4}{*}{ Bjor } & 0 & $\begin{array}{c}10.31 \pm 1.38 \mathrm{~g} \\
(100)\end{array}$ & $\begin{array}{c}2.56 \pm 0.41 \mathrm{f} \\
(100)\end{array}$ & $\begin{array}{c}120 \pm 30 \mathrm{c} \\
(100)\end{array}$ & $4.03 \mathrm{ae}$ & $0.086 \mathrm{~cd}$ \\
\hline & 50 & $\begin{array}{c}9.45 \pm 1.56 \mathrm{df} \\
(91.6)\end{array}$ & $\begin{array}{c}2.04 \pm 0.14 a \\
(79.7)\end{array}$ & $\begin{array}{c}105 \pm 43 \mathrm{ab} \\
(87.5)\end{array}$ & $4.63 \mathrm{~b}$ & $0.090 \mathrm{c}$ \\
\hline & 250 & $\begin{array}{c}7.12 \pm 2.11 \mathrm{ac} \\
(69.1)\end{array}$ & $\begin{array}{c}1.66 \pm 0.31 d \\
(64.8)\end{array}$ & $\begin{array}{c}105 \pm 56 \mathrm{ab} \\
(87.5)\end{array}$ & $4.29 \mathrm{e}$ & $0.068 \mathrm{ab}$ \\
\hline & 650 & $\begin{array}{c}5.56 \pm 0.97 \text { be } \\
(53.9)\end{array}$ & $\begin{array}{c}0.83 \pm 0.11 \mathrm{~b} \\
(32.4)\end{array}$ & $\begin{array}{c}90 \pm 45 \mathrm{~d} \\
(75.0)\end{array}$ & $6.70 \mathrm{~g}$ & $0.062 a b$ \\
\hline \multirow{4}{*}{ Jorr } & 0 & $\begin{array}{c}8.39 \pm 2.34 \mathrm{a} \\
(100)\end{array}$ & $\begin{array}{c}2.23 \pm 0.75 \mathrm{e} \\
(100)\end{array}$ & $\begin{array}{c}130 \pm 42 \mathrm{e} \\
(100)\end{array}$ & $3.76 \mathrm{a}$ & $0.064 a b$ \\
\hline & 50 & $\begin{array}{c}7.50 \pm 2.02 \mathrm{ad} \\
(89.4)\end{array}$ & $\begin{array}{c}2.03 \pm 0.21 \mathrm{a} \\
(91.0)\end{array}$ & $\begin{array}{c}110 \pm 53 \mathrm{ac} \\
(84.6)\end{array}$ & $3.69 \mathrm{a}$ & $0.068 \mathrm{ab}$ \\
\hline & 250 & $\begin{array}{c}6.78 \pm 1.73 b c \\
(80.8)\end{array}$ & $\begin{array}{c}1.40 \pm 0.17 \mathrm{c} \\
(62.8)\end{array}$ & $\begin{array}{c}105 \pm 40 \mathrm{ab} \\
(80.8)\end{array}$ & $4.84 \mathrm{bf}$ & $0.064 a b$ \\
\hline & 650 & $\begin{array}{c}5.15 \pm 2.08 \mathrm{e} \\
(61.4)\end{array}$ & $\begin{array}{c}0.93 \pm 0.20 \mathrm{~b} \\
(41.7)\end{array}$ & $\begin{array}{c}90 \pm 35 \mathrm{~d} \\
(69.2)\end{array}$ & $5.54 \mathrm{~d}$ & $0.057 \mathrm{a}$ \\
\hline \multirow{4}{*}{ Tora } & 0 & $\begin{array}{c}12.72 \pm 2.05 \mathrm{~h} \\
(100)\end{array}$ & $\begin{array}{c}2.43 \pm 0.83 f \\
(100)\end{array}$ & $\begin{array}{c}135 \pm 35 \mathrm{e} \\
(100)\end{array}$ & $5.23 \mathrm{~cd}$ & $0.094 \mathrm{c}$ \\
\hline & 50 & $\begin{array}{c}10.85 \pm 2.14 \mathrm{fg} \\
(85.3)\end{array}$ & $\begin{array}{c}2.11 \pm 0.54 \mathrm{ae} \\
(86.8)\end{array}$ & $\begin{array}{c}115 \pm 44 \text { ac } \\
(85.2)\end{array}$ & $5.14 \mathrm{cf}$ & $0.094 \mathrm{c}$ \\
\hline & 250 & $\begin{array}{c}8.27 \pm 0.87 \mathrm{ad} \\
(65.0)\end{array}$ & $\begin{array}{c}1.73 \pm 0.19 d \\
(71.2)\end{array}$ & $\begin{array}{c}112 \pm 45 \text { ac } \\
(82.9)\end{array}$ & $4.78 \mathrm{~b}$ & $0.073 \mathrm{bd}$ \\
\hline & 650 & $\begin{array}{c}6.74 \pm 0.29 \mathrm{bc} \\
(52.9)\end{array}$ & $\begin{array}{c}1.25 \pm 0.12 \mathrm{c} \\
(51.4)\end{array}$ & $\begin{array}{c}98 \pm 40 \mathrm{bd} \\
(72.6)\end{array}$ & $5.39 \mathrm{~cd}$ & $0.068 a b$ \\
\hline \multicolumn{7}{|c|}{ Means for primary effects - Średnie dla efektów głównych } \\
\hline \multirow{3}{*}{$\begin{array}{l}\text { Varieties } \\
\text { Odmiana }\end{array}$} & Bjor & $8.11 \mathrm{ab}$ & $1.77 \mathrm{a}$ & $105 \mathrm{a}$ & $4.91 \mathrm{a}$ & 0.076 a \\
\hline & Jorr & $6.96 \mathrm{a}$ & $1.65 \mathrm{a}$ & 109 a & $4.46 \mathrm{a}$ & $0.063 \mathrm{~b}$ \\
\hline & Tora & $9.65 \mathrm{~b}$ & $1.88 \mathrm{a}$ & 115 a & $5.13 \mathrm{a}$ & $0.082 \mathrm{a}$ \\
\hline \multirow{4}{*}{$\begin{array}{l}\text { Dose } \\
\text { Dawka }\end{array}$} & 0 & $10.47 \mathrm{a}$ & $2.41 \mathrm{~d}$ & $128 \mathrm{c}$ & $4.34 \mathrm{a}$ & $0.081 \mathrm{~b}$ \\
\hline & 50 & $9.27 \mathrm{a}$ & $2.06 \mathrm{c}$ & $110 \mathrm{a}$ & $4.49 \mathrm{a}$ & $0.084 \mathrm{~b}$ \\
\hline & 250 & $7.39 \mathrm{c}$ & $1.60 \mathrm{~b}$ & 107 a & $4.64 \mathrm{a}$ & $0.068 \mathrm{a}$ \\
\hline & 650 & $6.36 \mathrm{~b}$ & $1.00 \mathrm{a}$ & $93 \mathrm{~b}$ & $5.88 \mathrm{~b}$ & $0.062 \mathrm{a}$ \\
\hline
\end{tabular}

a-i - homogeneous groups according to the Duncana test, $\alpha<0.05$ - grupy jednorodne wyznaczone przy użyciu testu Duncana, $\alpha<0,05$. 
Transpiration rate of the basket willow cultivars being described differed depending on the cultivar and the zinc salt dose being used to nutrient solution. The intensity of transpiration in the examined basket willow cultivars ranged from 0.83 to $2.56\left(\mathrm{mmol} \mathrm{H} \mathrm{H}_{2} \mathrm{O} \cdot \mathrm{m}^{-2} \cdot \mathrm{s}^{-1}\right)$ depending from the zinc salt dose being used to nutrient solution. The zinc salt dose of $650 \mathrm{mg} \cdot \mathrm{dm}^{-3}$ being used induced reduction in transpiration rate in cultivars Bjor, Jorr and Tora by $67.6 \%, 58.3 \%$ and $48.6 \%$, respectively, in relation to the control plants (Table 2 ). The highest intensity of this process was characteristic of the cultivar Tora, while the lowest one of the cultivar Jorr. No statistically significant differences were found in transpiration rate between the cultivars being examined (Table 2).

The study conducted by Malinowska et al. $(2010 \mathrm{~b}, 2015)$ on the activity of assimilation and transpiration in the described basket willow cultivars growing in a nutrient solution contaminated with lead salt has shown significant differences in the activity of these processes between the cultivars, indicating a higher rate of the two processes in cultivar Jorr compared to cultivars Bjor and Tora. On the other hand, the study conducted by Malinowska and Wróbel (2015) on the activity of assimilation and transpiration in the basket willow cultivars being described has shown a higher rate of these processes un cultivar Tora compared to cultivars Bjor and Jorr.

From among the basket willow cultivars being examined, cultivar Tora was characterised by the highest stomatal conductance. The highest dose of zinc salt being used in nutrient solution induced reduction in stomatal conductance in cultivars Bjor, Jorr and Tora by $25.0 \%$, $30.8 \%$ and $27.4 \%$, respectively, in relation to the cultivars growing in complete nutrient solution (Table 2). Many authors have emphasised that stomatal conductance is regulated largely by carbon dioxide concentration in the intracellular spaces, leaf water potential and interaction of many environmental factors, among others, increased content of heavy metals in substrate (Kefu et al. 1991; Maier-Maercker and Koch 1992; Sala and Tenhunen 1994; Mott and Buckley 2000; Tuzet et al. 2003).

Assimilation and transpiration rates depend largely on stomatal conductance which determines the rate of diffusion of water vapour out of the leaf and the linear velocity of forced air flow through the leaf (Ludlow 1982). In the basket willow cultivars being examined, significant positive correlations between assimilation, transpiration and stomatal conductance were found (Table 3). Significant linear correlations between assimilation and transpiration rates and stomatal conductance in the trees growing in the urban environment have been obtained by Malinowska (2012). Wróbel and Gregorczyk (2004) and Wróbel et al. (2006) have shown a linear correlation between transpiration and stomatal conductance in the basket willow growing under stress conditions. Mikiciuk and Mikiciuk (2009) have found, as a result of their study, a significant linear correlation between stomatal conductance and transpiration in the strawberry cultivar Elvira. In the study conducted by Malinowska and Wróbel (2015), $\mathrm{CO}_{2}$ assimilation and transpiration of the basket willow growing in a contaminated substrate have been significantly reduced by stomatal conductance.

Stomatal closure is a response of plants to many stressors and follows a complicated mechanism that is associated with phosphorylation of certain proteins, increased content of abscisic acid, which leads to $\mathrm{H}_{2} \mathrm{O}_{2}$ accumulation and activation of calcium channels in the membranes of guard cells, resulting in the reduction of transpiration rate (Mott and Parkhurst 1991; Hinckley and Braatne 1994; Laloi et al. 2004). 
Table 3. Values of coefficients of correlation between the parameters of gas exchange and stomatal conductance of basket willow

Tabela 3. Wartości współczynników korelacji między badanymi parametrami wymiany gazowej a przewodnością szparkową u wierzby wiciowej

\begin{tabular}{|c|c|c|c|}
\hline $\begin{array}{l}\text { Variety } \\
\text { Odmiana }\end{array}$ & $\begin{array}{l}\text { Parameters } \\
\text { Parametry }\end{array}$ & $\begin{array}{c}\text { Parameters } \\
\text { Parametry }\end{array}$ & $\begin{array}{c}\text { Correlation coefficients } \\
\text { Współczynnik korelacji (r) }\end{array}$ \\
\hline \multicolumn{4}{|c|}{ Dose of $\mathrm{Zn}-$ dawka $\mathrm{Zn}: 0 \mathrm{mg} \cdot \mathrm{dm}^{-3}$} \\
\hline \multirow{2}{*}{ Bjor } & $\begin{array}{l}\mathrm{CO}_{2} \text { assimilation } \\
\text { asymilacja } \mathrm{CO}_{2}\end{array}$ & $\begin{array}{l}\text { stomatal conductance } \\
\text { przewodność szparkowa }\end{array}$ & 0.356 \\
\hline & $\begin{array}{l}\text { transpiration } \\
\text { transpiracja }\end{array}$ & $\begin{array}{l}\text { stomatal conductance } \\
\text { przewodność szparkowa }\end{array}$ & $0.481^{*}$ \\
\hline \multirow{2}{*}{ Jorr } & $\begin{array}{l}\mathrm{CO}_{2} \text { assimilation } \\
\text { asymilacja } \mathrm{CO}_{2}\end{array}$ & $\begin{array}{l}\text { stomatal conductance } \\
\text { przewodność szparkowa }\end{array}$ & 0.409 \\
\hline & $\begin{array}{l}\text { transpiration } \\
\text { transpiracja }\end{array}$ & $\begin{array}{l}\text { stomatal conductance } \\
\text { przewodność szparkowa }\end{array}$ & $0.532^{*}$ \\
\hline \multirow{2}{*}{ Tora } & $\begin{array}{l}\mathrm{CO}_{2} \text { assimilation } \\
\text { asymilacja } \mathrm{CO}_{2}\end{array}$ & $\begin{array}{l}\text { stomatal conductance } \\
\text { przewodność szparkowa }\end{array}$ & $0.463^{*}$ \\
\hline & $\begin{array}{l}\text { transpiration } \\
\text { transpiracja }\end{array}$ & $\begin{array}{l}\text { stomatal conductance } \\
\text { przewodność szparkowa }\end{array}$ & $0.498^{*}$ \\
\hline \multicolumn{4}{|c|}{ Dose of $\mathrm{Zn}$ - dawka $\mathrm{Zn}: 50 \mathrm{mg} \cdot \mathrm{dm}^{-3}$} \\
\hline \multirow{2}{*}{ Bjor } & $\begin{array}{l}\mathrm{CO}_{2} \text { assimilation } \\
\text { asymilacja } \mathrm{CO}_{2}\end{array}$ & $\begin{array}{l}\text { stomatal conductance } \\
\text { przewodność szparkowa }\end{array}$ & 0.412 \\
\hline & $\begin{array}{l}\text { transpiration } \\
\text { transpiracja }\end{array}$ & $\begin{array}{l}\text { stomatal conductance } \\
\text { przewodność szparkowa }\end{array}$ & $0.535^{\star}$ \\
\hline \multirow{2}{*}{ Jorr } & $\begin{array}{l}\mathrm{CO}_{2} \text { assimilation } \\
\text { asymilacja } \mathrm{CO}_{2}\end{array}$ & $\begin{array}{l}\text { stomatal conductance } \\
\text { przewodność szparkowa }\end{array}$ & $0.487^{*}$ \\
\hline & $\begin{array}{l}\text { transpiration } \\
\text { transpiracja }\end{array}$ & $\begin{array}{l}\text { stomatal conductance } \\
\text { przewodność szparkowa }\end{array}$ & $0.568^{*}$ \\
\hline \multirow{2}{*}{ Tora } & $\begin{array}{l}\mathrm{CO}_{2} \text { assimilation } \\
\text { asymilacja } \mathrm{CO}_{2}\end{array}$ & $\begin{array}{l}\text { stomatal conductance } \\
\text { przewodność szparkowa }\end{array}$ & $0.524^{*}$ \\
\hline & $\begin{array}{l}\text { transpiration } \\
\text { transpiracja }\end{array}$ & $\begin{array}{l}\text { stomatal conductance } \\
\text { przewodność szparkowa }\end{array}$ & $0.586^{*}$ \\
\hline \multicolumn{4}{|c|}{ Dose of Zn - dawka Zn: $250 \mathrm{mg} \cdot \mathrm{dm}^{-3}$} \\
\hline \multirow{2}{*}{ Bjor } & $\begin{array}{l}\mathrm{CO}_{2} \text { assimilation } \\
\text { asymilacja } \mathrm{CO}_{2}\end{array}$ & $\begin{array}{l}\text { stomatal conductance } \\
\text { przewodność szparkowa }\end{array}$ & $0.611^{*}$ \\
\hline & $\begin{array}{l}\text { transpiration } \\
\text { transpiracja }\end{array}$ & $\begin{array}{l}\text { stomatal conductance } \\
\text { przewodność szparkowa }\end{array}$ & $0.583^{*}$ \\
\hline \multirow{2}{*}{ Jorr } & $\begin{array}{l}\mathrm{CO}_{2} \text { assimilation } \\
\text { asymilacja } \mathrm{CO}_{2}\end{array}$ & $\begin{array}{l}\text { stomatal conductance } \\
\text { przewodność szparkowa }\end{array}$ & $0.684^{*}$ \\
\hline & $\begin{array}{l}\text { transpiration } \\
\text { transpiracja }\end{array}$ & $\begin{array}{l}\text { stomatal conductance } \\
\text { przewodność szparkowa }\end{array}$ & $0.738^{*}$ \\
\hline \multirow{2}{*}{ Tora } & $\begin{array}{l}\mathrm{CO}_{2} \text { assimilation } \\
\text { asymilacja } \mathrm{CO}_{2}\end{array}$ & $\begin{array}{l}\text { stomatal conductance } \\
\text { przewodność szparkowa }\end{array}$ & $0.667^{*}$ \\
\hline & $\begin{array}{l}\text { transpiration } \\
\text { transpiracja }\end{array}$ & $\begin{array}{l}\text { stomatal conductance } \\
\text { przewodność szparkowa }\end{array}$ & $0.729^{*}$ \\
\hline \multicolumn{4}{|c|}{ Dose of Zn - dawka Zn: $650 \mathrm{mg} \cdot \mathrm{dm}^{-3}$} \\
\hline \multirow{2}{*}{ Bjor } & $\begin{array}{l}\mathrm{CO}_{2} \text { assimilation } \\
\text { asymilacja } \mathrm{CO}_{2}\end{array}$ & $\begin{array}{l}\text { stomatal conductance } \\
\text { przewodność szparkowa }\end{array}$ & $0.558^{*}$ \\
\hline & $\begin{array}{l}\text { transpiration } \\
\text { transpiracja } \\
\end{array}$ & $\begin{array}{l}\text { stomatal conductance } \\
\text { przewodność szparkowa }\end{array}$ & $0.643^{*}$ \\
\hline \multirow{2}{*}{ Jorr } & $\begin{array}{l}\mathrm{CO}_{2} \text { assimilation } \\
\text { asymilacja } \mathrm{CO}_{2} \\
\end{array}$ & $\begin{array}{l}\text { stomatal conductance } \\
\text { przewodność szparkowa }\end{array}$ & $0.727^{\star}$ \\
\hline & $\begin{array}{l}\text { transpiration } \\
\text { transpiracja }\end{array}$ & $\begin{array}{l}\text { stomatal conductance } \\
\text { przewodność szparkowa }\end{array}$ & $0.841^{*}$ \\
\hline \multirow{2}{*}{ Tora } & $\begin{array}{l}\mathrm{CO}_{2} \text { assimilation } \\
\text { asymilacja } \mathrm{CO}_{2}\end{array}$ & $\begin{array}{l}\text { stomatal conductance } \\
\text { przewodność szparkowa }\end{array}$ & $0.806^{*}$ \\
\hline & $\begin{array}{l}\text { transpiration } \\
\text { transpiracja }\end{array}$ & $\begin{array}{l}\text { stomatal conductance } \\
\text { przewodność szparkowa }\end{array}$ & $0.876^{*}$ \\
\hline
\end{tabular}

${ }^{*}$ significant at $\alpha=0.05$ - istotny na poziomie istotności $\alpha=0,05$. 
Photosynthetic water-use efficiency is often a decisive indicator of plant productivity under stress conditions (Garczyński 2004; Górny and Garczyński 2002). The calculated rate varied, depending on the zinc salt doses being used. Statistical analysis did not show any differences in the value of the photosynthetic rate of water use between the basket willow cultivars being examined. Cultivar Tora used water slightly better in the photosynthesis process than cultivars Bjor and Jorr (Table 2). Wróbel (2006) and Wróbel et al. (2006) Malinowska and Wróbel (2015), Wróbel and Wróbel (2015) have found in their studies conducted on the basket willow growing on a degraded substrate that cultivar Jorr was characterised by a significantly higher WUE value.

In the carried out study, the average values of water use rates for the examined basket willow cultivars ranged from 3.69 to 6.70 for WUE, and from 0.057 to 0.094 for WUEI (Table 2). Significant differences in the value of WUE and WUEI were obtained, depending on the zinc salt dose used. The examined basket willow cultivars growing in nutrient solution with the the highest dose of zinc salt were characterised by significantly higher values of WUE (Table 2). Jaroszewska et al. (2011) have showed higher values of the photosynthetic rate of water-use efficiency for the cherry tree growing under water and nutrient stress conditions compared to the control conditions. Olszewska and Gregorczyk (2013) have obtained similar relationships for different grass species exposed to water stress.

The observed reduction (even by $50 \%$ ) in the values of physiological parameters, i.e. $\mathrm{CO}_{2}$ assimilation, transpiration and stomatal conductance, as well as photosynthetic pigments under the conditions of substrate contamination with zinc, compared to the control object, allows the examined Salix viminalis L. cultivars to maintain their vital functions during the period of stress impact. This suggests their special predispositions for phytoremediation.

The results obtained for the physiological parameters being analysed may be useful for assessing the resistance of the examined basket willow cultivars to the stress induced by increased amount of zinc ions and their suitability for phytoremediation.

\section{CONCLUSIONS}

1. Gas exchange parameters, content of photosynthetic pigments and water balance in the examined basket willow cultivars were significantly differentiated by the dose of zinc salt in nutrient solution.

2. Net assimilation and transpiration in the examined basket willow cultivars growing in nutrient solution with zinc ions added were significantly reduced by stomatal conductance.

3. The examined basket willow cultivars growing in nutrient solution with the highest dose of zinc salt were characterised by significantly higher values of the photosynthetic rate of water use.

4. Increasing doses of zinc salt in nutrient solution induced reduction in the content of photosynthetic pigments in leaves of the examined basket willow cultivars.

5. A quite high physiological activity being found in three examined basket willow cultivars under the conditions of substrate contamination with zinc suggests their suitability for phytoremediation, with cultivar Tora distinguishing itself in this respect from among the examined cultivars. 


\section{REFERENCES}

Arnon D.J., Allen M.B., Whatley F. 1956. Photosynthesis by isolated chloroplast. IV General concept and comparison of three photochemical reactions. Biochim. Biophys. Acta 20, 449-461.

Bandurska H. 1991. The effect of proline on nitrate reductase activity in water - stressed baryle leaves. Acta Physiol. Plant. 1, 3-11.

Baran A., Jasiewicz C. 2009. Toksyczna zawartość cynku i kadmu w glebie dla różnych gatunków roślin [The toxicity content of zinc and cadmium in soil to different plant species]. Ochr. Środ. Zasob. Natur. 40, 157-164. [in Polish]

Baran S. 2000. Ocena stanu degradacji i rekultywacji gleb. Lublin, Wydaw. AR, 225. [in Polish]

Garczyński S. 2004. Wpływ genotypu i poziomu nawożenia na zmienność w fotosyntetycznej aktywności najwyższych liści u pszenicy tetraploidalnej [The effect of genotype and fertilisation level on variation in the photosynthetic activity of the highest leaves in tetraploid wheat]. Zesz. Probl. Post. Nauk. Rol. 496, 357-366. [in Polish]

Górny A.G., Garczyński S. 2002. Genotypic and nutrition-dependent variation in water use efficiency and photosynthetic activity of leaves in winter wheat (Triticum aesativum L.). J. Appl. Genet. 43(2), 145-160.

Gruca-Królikowska S., Wacławek W. 2006. Metale w środowisku. Cz. II. Wpływ metali ciężkich na środowisko [Metals in the environment. Part II. Impact of heavy metals on the environment]. Chem. Dydak. Ekol. Metrol. 11(1-2), 41-56. [in Polish]

Hager A., Mayer-Berthenrath T. 1966. Die Isolierung und quantitative Bestimmung der Carotinoide und Chlorophyll von Blatern, Algen und isolierten Chloroplasten mit Hilfe Dunnschicht-chromatographischer Methoden [Isolation and quantitative determination of carotenoids and chlorophylls of leaves, algae and isolated chloroplasts using thin-layer chromatographic methods]. Planta, Berlin 69, 198-217. [in Germany]

Hinckly T.M., Braatne J.H. 1994. Stomata, in: Plant - environment interaction. Red. R. Wilkinson, K. Marcel. New York, Dekker Inc., 323-355.

Jaroszewska A., Podsiadło C., Kowalewska R. 2011. Analiza wykorzystania wody przez wiśnię w różnych warunkach wodnych i nawozowych [Analysis of the water use by the cherry tree under different water and fertilisation conditions]. Infr. Ekol. Ter. Wiej. 6, 165-173. [in Polish]

Kefu Z., Munns R., King R.W. 1991. Abscisic acid levels in NaCl-treated barley, cotton and saltbush. Aust. J. Plant Physiol. 18, 17-24.

Kabata-Pendias A., Pendias H. 1999. Biogeochemia pierwiastków śladowych. Warszawa, PWN. [in Polish]

Kostrzewska-Kuczumow J., Weryszko-Chmielewska E. 2000a. Reakcja trzech gatunków roślin uprawnych na nadmierne stężenia cynku [Differentiated sensitivity of some cultivated plant species to zinc excess]. Biul. Magnezol. 5(4), 297-303. [in Polish]

Kostrzewska-Kuczumow J., Weryszko-Chmielewska E. 2000b. Zmiany histologiczne w łodygach grochu (Pisum sativum L.) w warunkach nadmiaru cynku [Histopathological changes in the stems of pea (Pisum sativum L.) under zinc excess conditions]. Biul. Magnezol. 5(1), 30-36. [in Polish]

Laloi C., Apel K., Danon A. 2004. Reactive oxygen signaling: the latest news. Curr. Opin. Plant Biol. 7, 323-328.

Landberg T., Greger M. 1996. Differences in uptake and tolerance to heavy metals in Salix from unpolluted and polluted areas. Appl. Geochem. 11, 175-180.

Laureysens I., Blust R., De Temmerman L., Lemmens C., Ceulemans R. 2004. Clonal variation in heavy metal accumulation and biomass production in a poplar coppice culture. I. Seasonal variation in leaf, wood and bark concentrations. Environ. Poll. 131, 485-494.

Laureysens I., De Temmerman L., Hastir T., Van Gysel M., Ceulemans R. 2005. Clonal variation in heavy metal accumulation and biomass production in a poplar coppice culture. II. Vertical distribution and phytoextraction potential. Environ. Poll. 133, 541-551. 
Lichtenthaler H.K. 1987. Chlorophylls and carotenoids: Pigments of photosynthetic biomembranes. Methods Enzymol. 148, 350-380.

Ludlow M.M. 1982. Measurements of stomatal conductance and plant water status, in: Techniques in bioproductivity and photosynthesis. Eds. J. Coombs, D.O. Oxford, Hall, Pergamon Press, 44-57.

Maier-Maercker U., Koch W. 1992. The effect of air pollution on the mechanism of stomatal control. Trees 7(1), 12-25.

Malinowska K., Mikiciuk M., Berdzik A. 2010a. Zmiany wybranych parametrów fizjologicznych wierzby wiciowej (Salix viminalis L.) wywołane zróżnicowanym stężeniem kadmu w podłożu [Changes of selected physiological parameters of basket willow (Salix viminalis L.) caused by a differentiated concentration of cadmium in the medium]. Ochr. Środ. Zas. Natur. 42, 24-32. [in Polish]

Malinowska K., Wróbel J., Malinowski R., Stera A. 2010b. The toxic impact of lead on the physiological parameters of basket willow (Salix viminalis L.). Agricult. Sci., Plovdiv 2(4), 45-49.

Malinowska K., Mikiciuk M., Wróbel J., Czyżyk E. 2011. Influence of cadmium on physiological parameters of clone Jorr of basket willow (Salix viminalis L.) from aquatic cultures. Ecol. Chem. Eng. A 18(5-6), 763-770.

Malinowska K. 2012. Oddziaływanie warunków miejskich na niektóre parametry fizjologiczne wybranych gatunków drzew w Szczecinie. Szczecin, Wydaw. ZUT, 120. [in Polish]

Malinowska K., Wróbel J. 2015. Physiological activity of common osier (Salix viminalis L.) under copper-induced stress conditions. Acta Agrophys. 22(2), 173-182.

Malinowska K., Malinowski R., Stera A. 2015. Physiological reaction of common osier (Salix viminialis L.) var. Jorr to the presence of lead in the subsoil. J. Ecol. Eng. 16(2), 110-115.

Mikiciuk G., Mikiciuk M. 2009. Wpływ dolistnego nawożenia potasowo-krzemowego na wybrane cechy fizjologiczne truskawki (Fragaria ananasa Duch.) odmiany Elvira [The influence of foliar application of potassium and silicon fertilizer on some physiological features of strawberry (Fragaria ananassa Duch.) variety Elvira]. Ann. UMCS 64(4), 19-27. [in Polish]

Mott K.A., Parkhurst D.F. 1991. Stomatal response to humidity in Ir and helom. Plant Cell Environ. 14, 509-515.

Mott K.A., Buckley T.N. 2000. Patchy stomatal conductance: emergent collective behaviour of stomata. Trends Plant Sci. 6, 258-262.

Olszewska M., Grzegorczyk S. 2013. Oddziaływanie stresu wodnego na wybrane gatunki traw uprawianych na glebie organicznej [The effect of water stress on selected grass species grown in organic soils]. Fragm. Agron. 30(3), 140-147. [in Polish]

Sala A., Tenhunen J.D. 1994. Site-specific water relations and stomatal response of Quercus ilex in a Mediterranean watershed. Tree Physiol. 14(6), 601-617.

Szatanik-Kloc A., Sokołowska Z., Hajnos M., Alekseeva T., Alekseev A. 2010. Wpływ jonów $\mathrm{Cu}^{+2}$ i $\mathrm{Zn}^{+2}$ na zawartość wapnia w życie (Secale cereale L.) [Effect $\mathrm{pH}$ and $\mathrm{Cu}^{+2}$ and $\mathrm{Zn}^{+2}$ ions on concentration of calcium in rye (Secale cereale L.)]. Acta Agrophys. 15(1), 177-185. [in Polish]

Szatanik-Kloc A., Bowanko G. 2007. Wpływ pH i jonów cynku na powierzchnię właściwą korzeni żyta (Secale cereale L.) wyznaczaną metodą adsorpcji-desorpcji azotu [Effect of pH and zinc on Surface area of rye (Secale cereale L.) roots as determined from nitrogen adsorption-desorption data]. Acta Agrophys. 10(3), 705-713. [in Polish]

Szczukowski S., Tworkowski J., Stolarski M. 2004. Wierzba energetyczna. Kraków, Wydaw. Plantpress, 46. [in Polish]

Tuzet A., Perrier A., Leuning R. 2003. A coupled model of stomatal conductance, photosynthesis and transpiration. Plant Cell. Environ. 26, 1097-1112.

Wróbel J., Gregorczyk A. 2004. Wstępne badania tolerancji trzech form Salix viminalis L. na zróżnicowane stężenie $\mathrm{NaCl}$ wprowadzone do podłoża [Preliminary studies of the tolerance of three forms of Salix viminalis $\mathrm{L}$. to different $\mathrm{NaCl}$ concentrations added to substrate]. Zesz. Probl. Post. Nauk Rol. 496, 403-413. [in Polish]

Wróbel J. 2006. Kinetyka wzrostu oraz wybrane wskaźniki fizjologiczne Salix viminalis uprawianej na refulacie piaszczystym nawożonym osadem ściekowym [Growth kinetics and selected physiological parameters of Salix viminalis cultivated on dredged sandy material fertilised with sewage sludge]. Rozpr. AR Szczec. 239, 133. [in Polish] 
Wróbel J., Mikiciuk M., Stolarska A. 2006. Wpływ warunków zasolenia gleby na aktywność wymiany gazowej u trzech klonów wierzby wiciowej (Salix viminalis L.) [The effect of soil salinity conditions on the activity of gas exchange in three common osier (Salix viminalis L.) clones]. Zesz. Probl. Post. Nauk Rol. 509, 269-281. [in Polish]

Wróbel J., Wróbel M. 2015. Porównanie parametrów aktywności wymiany gazowej oraz plonu trzech odmian wierzby wiciowej (Salix viminalis L.) pochodzących z wieloletniej plantacji [Comparison of parameters of gas exchange activity and yields of three varieties of common osier (Salix viminalis L.) from a multi-year plantation]. Acta Agrophys. 22(2), 219-231. [in Polish]

Wrzosek J., Gawroński S., Gworek B. 2008. Zastosowanie roślin energetycznych w technologii fitoremediacji [The use of crop plants cultivated for energy in phytoremediation]. Ochr. Środ. Zas. Natur. 37, 139-151 [in Polish].

Zabrocki R., Ignacek G. 2008. Wykorzystanie wierzby energetycznej w gospodarce rolnej [The use of Salix viminalis in agriculture]. Rocz. Nauk. Stow. Ekon. Rol. Agrobiz. 9(3), 234-238. [in Polish]

Zemleduch A., Tomaszewska B. 2007. Mechanizmy, procesy i oddziaływanie w fitoremediacji [Mechanisms, proceses and interactions during phytoremediation]. Kosmos Probl. Nauk Biol. 56(3-4), 393-407. [in Polish]

Abstract. The influence of zinc salt, in a concentration range of 50 to $650 \mathrm{mg} \cdot \mathrm{dm}^{-3}$, on the physiological response of basket willow was studied on Bjor, Tora and Jorr varieties which were grown in hydroponics. Content of chloroplast pigments, relative water content (RWC), water saturation deficit (WSD), intensity of assimilation and transpiration, stomatal conductance, photosynthetic rate of water use efficiency (WUE) and instantaneous photosynthetic rate of water use (WUEI) were determined in leaves. It was observed that the decrease in concentration of tested physiological parameters correlated with the increase of zinc salt doses in the medium. The assimilation and transpiration of basket willow were significantly limited by stomata. The addition of zinc salt to the medium increased water saturation deficit in leaves of all three Salix viminalis verities. 
